\title{
Metamaterial with Negative Index due to Chirality
}

\author{
E. Plum, ${ }^{1, *}$ J. Zhou ${ }^{2,3}$ J. Dong, ${ }^{4,3}$ V. A. Fedotov,${ }^{1}$ T. Koschny, ${ }^{3,5}$ C. M. Soukoulis,${ }^{3,5}$ and N. I. Zheludev ${ }^{1}$ \\ ${ }^{1}$ Optoelectronics Research Centre, University of Southampton, SO17 1BJ, UK \\ ${ }^{2}$ Department of Electrical and Computer Engineering and Microelectronics \\ Research Center, Iowa State University, Ames, Iowa 50011, USA \\ ${ }^{3}$ Ames Laboratory and Department of Physics and Astronomy, Iowa State University, Ames, Iowa 50011, USA \\ ${ }^{4}$ Institute of Optical Fiber Commun. and Network Tech., Ningbo University, Ningbo 315211, China \\ ${ }^{5}$ Institute of Electronic Structure and Laser - Foundation for Research and Technology Hellas (FORTH), \\ and Department of Materials Science and Technology, University of Crete, Greece
}

(Dated: July 31, 2008)

\begin{abstract}
Recently it has been predicted that materials with exceptionally strong optical activity may also possess a negative refractive index, allowing the realization of superlenses for super-resolution imaging and data storage applications. Here we demonstrate experimentally and numerically that chirality-induced negative refraction is possible. Negative refraction due to 3D chirality is demonstrated for a bilayered metamaterial based on pairs of mutually twisted planar metal patterns in parallel planes, which also shows negative electric and magnetic responses and exceptionally strong optical activity and circular dichroism. Multi-layered forms of the metamaterial are found to be suitable for use as ultra-thin polarization rotators and circular polarizers for practical applications.
\end{abstract}

\section{INTRODUCTION}

Optical activity, which is the ability to rotate the plane of polarization of electromagnetic waves, has always been a phenomenon of great importance to many areas of science, including molecular biology, analytical chemistry, detection of life forms, optoelectronics and display applications. Optical activity exhibited by natural materials like quartz, however, is quite weak and limited to the optical spectral range. Consequently artificial gyrotropic structures are of interest for polarization control applications in microwave and opto-electronic devices ${ }^{1-4}$. Since Pendry and Tretyakov recently predicted that strong optical activity may also result in negative refraction ${ }^{5,6}$, artificial gyrotropic materials have started to attracted a lot of attention as potential candidates for achieving negative refraction ${ }^{7-11}$. With respect to the realization of negative refraction due to optical activity, however, little progress was made until very strong microwave gyrotropy was reported for a single pair of mutually twisted metal patterns in parallel planes ${ }^{12}$. Importantly a signature of circularly polarized backward waves was observed for this structure, indicating that metamaterials based on such mutually twisted metal patterns might have a negative index of refraction. Recently a scaled down metamaterial-version of this structure was shown to also possess exceptionally strong polarization rotary power in the optical part of the spectrum ${ }^{13}$.

In this paper we demonstrate experimentally and numerically that metamaterials based on multiple layers of mutually twisted planar metal patterns in parallel planes (Fig. 1) support a wealth of useful electromagnetic properties including giant optical activity and circular dichroism, strong negative electric and magnetic responses and negative refraction. Due to their 4 -fold rotational symmetry, circular polarization conversion in our 3D-chiral metamaterials is negligible and they have circularly polarized eigenstates. These facts are confirmed experimen-

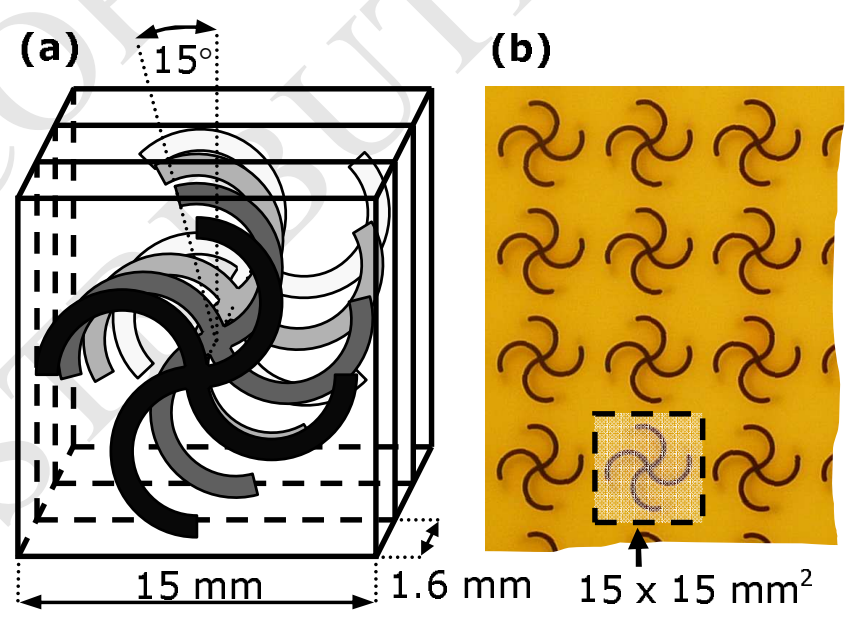

FIG. 1: Structure of the metamaterial. (a) Schematics of the 4-layered metamaterial's unit cell. The gammadions in neighboring layers have a relative twist of $15^{\circ}$. The structure of metamaterials with a different number of layers is analogous. (b) Photograph of part of a bilayered metamaterial sheet. The twisted gammadions of the $2^{\text {nd }}$ layer can be seen as a shaded area thanks to partial transparency to the substrate. A unit cell has been marked.

tally. Importantly we show for a bilayered metamaterial consisting of pairs of mutually twisted rosettes, that its negative refractive index arises from the structure's 3Dchiral symmetry. In contrast to conventional negative index materials, like split ring wire media, fishnet structures and double crosses $^{14-16}$, the negative index is not caused by simultaneous negative electric and magnetic responses. We also found that two layers of mutually twisted metal rosettes show strong polarization rotary power and circular dichroism and we study these effects by modeling how the polarization state changes as the wave travels through the metamaterial. Finally we find that multi-layered versions of the metamaterial, consist- 
ing of four or more layers of rosettes, lead to exceptionally strong optical activity and circular dichroism combined with reduced insertion losses, making such structures practical ultra-thin polarization rotators or circular polarizers.

\section{GIANT OPTICAL ACTIVITY AND CIRCULAR DICHROISM}

Fig. 2a shows transmission properties of the bilayered form of the metamaterial for left-handed (LCP) and right-handed (RCP) circular polarizations. The structure shows exceptionally strong circular dichroism of up to $20 \mathrm{~dB}$. For linear polarization, azimuth rotation of up to $25^{\circ}$ is achieved, however, in this case the transmitted polarization state becomes elliptical. Pure optical activity, i.e. polarization azimuth rotation without any change of ellipticity, is achieved between resonances $\mathbf{A}$ and $\mathbf{B}$, where the absolute rotation is about $7^{\circ}$. These values are substantial considering the material's thickness of only $1 / 30$ wavelength $\lambda$ at $6 \mathrm{GHz}$ where the strongest effects occur. In terms of rotation per material thickness of one wavelength the structure's peak rotary power and pure optical activity are $780^{\circ} / \lambda$ and $250^{\circ} / \lambda$ respectively. This is gigantic compared to naturally optically active crystals like quartz $\left(0.02^{\circ} / \lambda\right.$ at $\left.400 \mathrm{~nm}\right)$ in the visible part of the spectrum. The metamaterial also rotates several times stronger than helix-based artificial structures for microwaves $\left(156^{\circ} / \lambda^{17}\right)$. The metamaterial's exceptionally strong gyrotropic response is confirmed by numerical results (faint lines, Fig. 2), which are in excellent agreement with the experiments (dark lines). Our simulations allow us to determine the nature of the resonances A-D. The highly gyrotropic resonances $\mathrm{A}$ and $\mathrm{B}$, which will be discussed in detail in the following section, correspond to $\lambda / 2$ current modes, while the weaker high frequency resonances $\mathbf{C}$ and $\mathbf{D}$ have $3 \lambda / 2$ current modes.

\section{NEGATIVE REFRACTION DUE TO 3D CHIRALITY}

So far, negative refraction has been achieved in a variety of structures, from split ring wire media to fishnet designs and double crosses ${ }^{14-16}$. All of these structures were designed to superimpose electric and magnetic resonances, which separately drove the permittivity $\varepsilon$ and permeability $\mu$ negative in the same frequency region. While these achiral negative index materials have attracted a lot of attention, chiral negative index media have not been studied experimentally. This is surprising, since Pendry and Tretyakov predicted that negative refraction could be easier to achieve in chiral media $^{5,6}$. Importantly for chiral media the refractive index is $n_{ \pm}=\sqrt{\varepsilon \mu} \pm \kappa$, where '+' and '-' refer to the righthanded (RCP) and left-handed (LCP) circularly polarized eigenstates and $\kappa$ is the chirality parameter. This

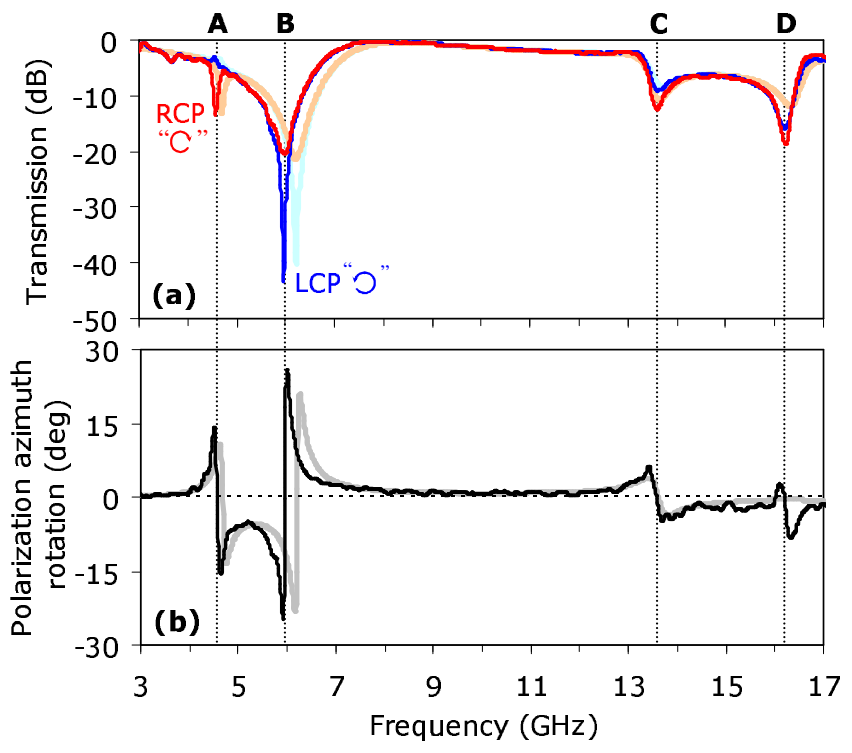

FIG. 2: Circular dichroism and optical activity of the bilayered metamaterial. Measurements (dark lines) and numerical simulations (faint lines) are shown. (a) Transmission levels for left-handed (blue, LCP, -) and right-handed (red, RCP, + ) circularly polarized waves. (b) Azimuth rotation for linearly polarized waves.

implies that in principle strong enough chirality is sufficient to achieve negative refraction for one circular polarization. The difficulty here is, that if $\sqrt{\varepsilon \mu}$ is not very close to zero, then very strong chirality is required indeed. Our bilayered metamaterial shows exceptionally strong gyrotropic behavior and thus it is an ideal candidate for negative refraction due to chirality.

Based on transmission and reflection we calculated the refractive index $n_{ \pm}$, chirality parameter $\kappa$, permeability $\mu$ and permittivity $\varepsilon$ (see appendix $\mathrm{C}$ ) for the bilayered 3Dchiral metamaterial and a reference structure in which $3 \mathrm{D}$ chirality has been removed by reducing the relative twist between paired rosettes to zero. The results, which are shown in Fig. 3, show that both chiral and achiral forms of the metamaterial have remarkably similar electric and magnetic responses. Particularly in both cases resonance $\mathbf{A}$ leads to negative permeability while resonance $\mathbf{B}$ corresponds to negative permittivity. The negative magnetic behavior for $\mathbf{A}$ results from anti-symmetric current oscillations in top and bottom rosettes, each pair of rosettes effectively forms a current loop, and thus a magnetic dipole (see Fig. $4 \mathbf{A}$ ). On the contrary the electric response for $\mathbf{B}$ results from in-phase current oscillations in pairs of rosettes, i.e. here pairs of rosettes act like a single electric dipole (see Fig. 4 B). The origin of the negative electric and magnetic responses of the bilayered structure is of the same nature as for fishnet structures and double crosses ${ }^{15,16}$.

In contrast to conventional negative index media, however, permeability and permittivity become negative in separate bands and $\sqrt{\varepsilon \mu}$ is always positive for both struc- 
(a)

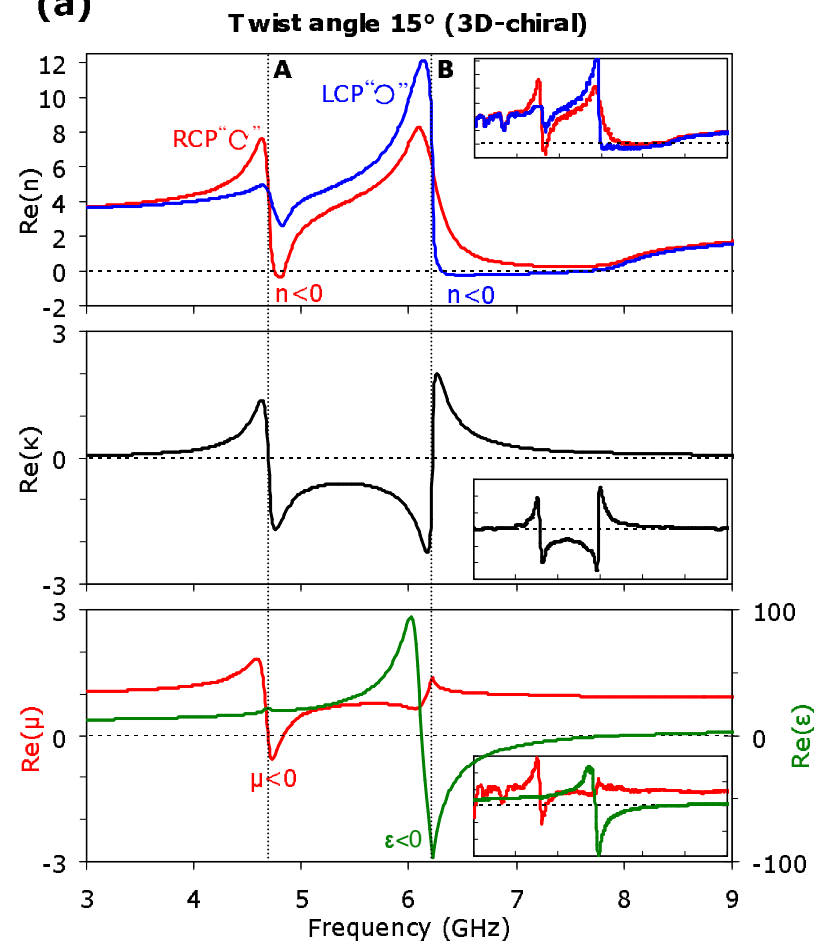

(b)

Twist angle $0^{\circ}$ (not 3D-chiral)
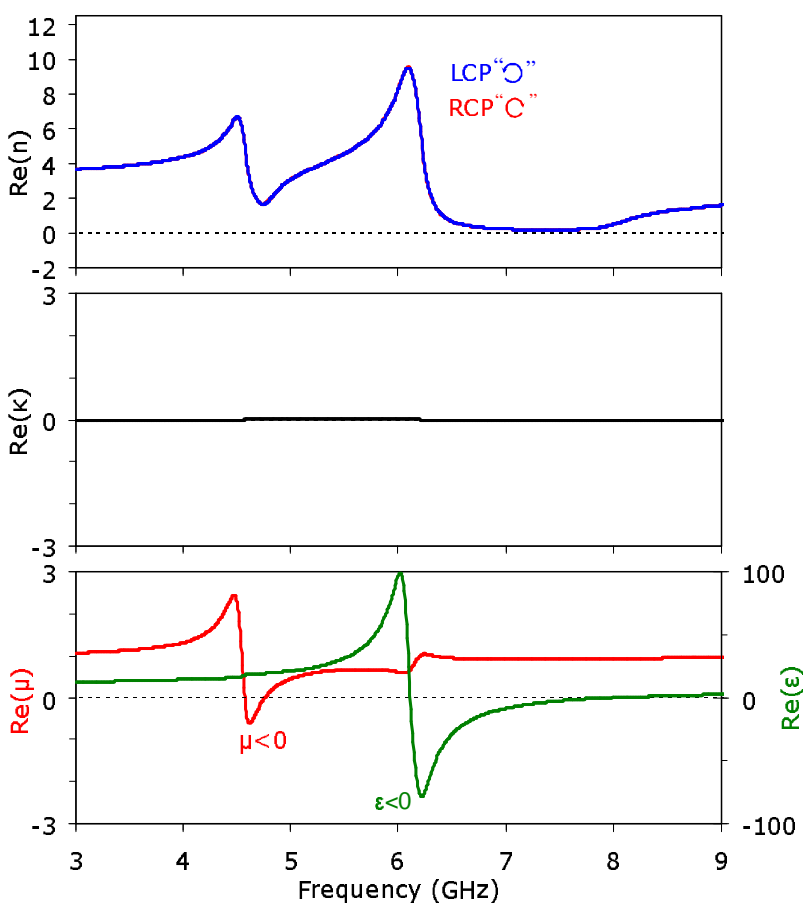

FIG. 3: (a) Effective medium parameters of the 3D-chiral bilayered metamaterial. Numerical results for refractive index $n$ (top), chirality parameter $\kappa$ (middle) and permeability $\mu$ and permittivity $\varepsilon$ (bottom) are shown. The insets show the corresponding experimental results with identical axis divisions. (b) Effective parameters derived from numerical simulations for a bilayered metamaterial with no relative twist between layers of rosettes. Note that $\varepsilon$ and $\mu$ are almost identical for both cases. Negative $n$ in the $3 \mathrm{D}$-chiral case arises from the contribution of the large chirality parameter $\kappa$.
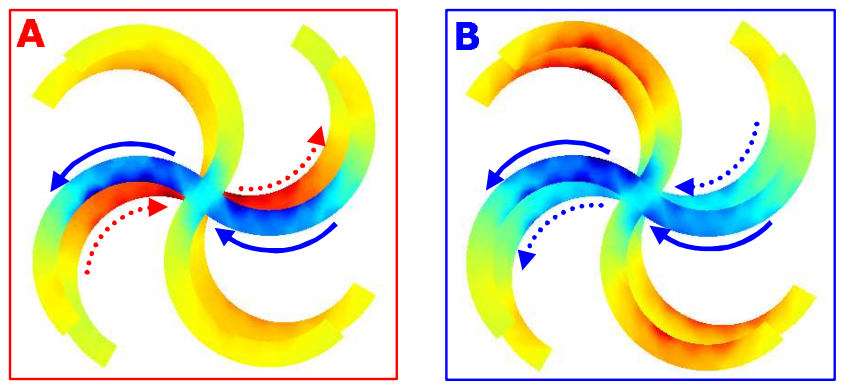

FIG. 4: Current modes leading to a negative refractive index. The anti-symmetric current mode $(\mathrm{A})$ is excited by RCP at $4.7 \mathrm{GHz}$ and the symmetric currentmode (B) is excited by LCP at $6.2 \mathrm{GHz}$. The horizontal component of the excited currents is shown, where blue and red correspond to currents in opposite directions.

tures. Nevertheless the 3D-chiral metamaterial has a negative refractive index just above resonances $\mathbf{A}$ (for RCP) and $\mathbf{B}$ (for LCP). Remarkably it is the large contribution from the chirality factor that drives the refractive index $n_{ \pm}=\sqrt{\varepsilon \mu} \pm \kappa$ negative. This is further illustrated by the fact, that the negative index disappears if $3 \mathrm{D}$ chirality is removed from the structure. Above resonance A a negative index of -0.4 is achieved due to the material's exceptionally strong gyrotropic response, while above resonance $\mathbf{B}$ a wide band of negative refraction can be achieved by moderate chirality as $\sqrt{\varepsilon \mu}$ is close to zero.

Also multi-layered forms of the metamaterial show signs of negative refraction. However, as these thicker structures cannot be assumed to be thin compared to the effective wavelength within the medium, effective parameter retrieval for multiple layers proves more difficult and lies beyond the scope of this paper.

\section{POLARIZATION STATE EVOLUTION WITHIN AN OPTICALLY ACTIVE METAMATERIAL}

We found that the bilayered 3D-chiral metamaterial shows exceptionally large circular dichroism and polarization rotary power sufficient to lead to a negative refractive index. The structure's highly gyrotropic response is a consequence of strong electromagnetic interaction between the mutually twisted wire patterns through the local field. The color-coded field maps shown in Fig. 5 present the evolution of the local field in terms of ellipticity (top) and azimuth (bottom) for the case of a linearly polarized wave propagating through the structure. The data corresponds to the frequency of $4.6 \mathrm{GHz}$, where the metamaterial rotates by $11^{\circ}$ and the transmitted wave 


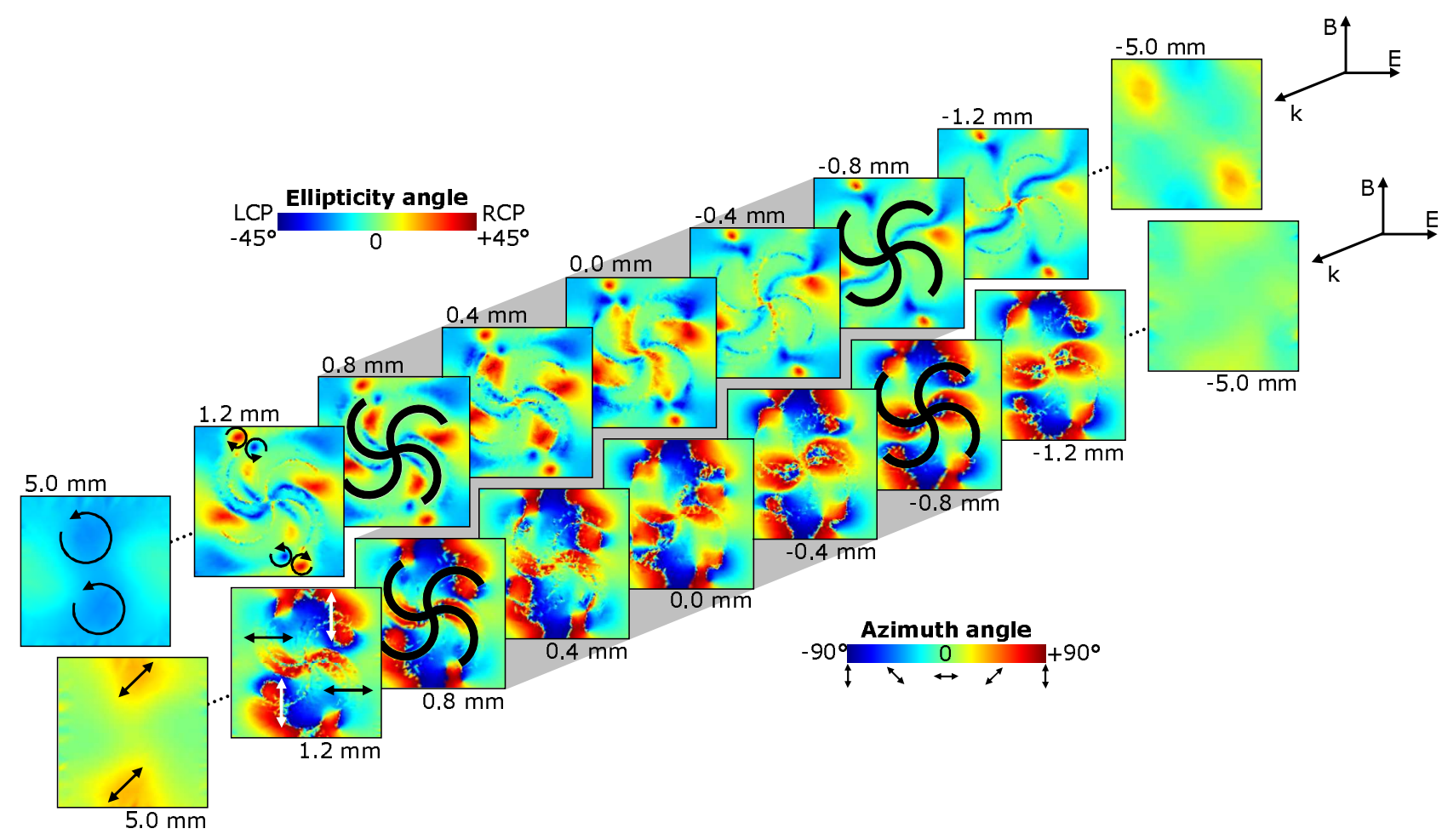

FIG. 5: Polarization state evolution for the total field as a linearly polarized wave propagates through the bilayered metamaterial at $4.6 \mathrm{GHz}$. The top sequence shows the ellipticity angle of the total field, while the bottom sequence shows the polarization azimuth.

has an ellipticity angle of $13^{\circ}$ (circular dichroism $4 \mathrm{~dB}$ ), while transmission is still reasonably large. Our simulations show that the incident linearly polarized plane wave is significantly perturbed by the metamaterial's structure within about $4 \mathrm{~mm}(\lambda / 16)$ of the material's surface. However, changes of the field's effective polarization state occur only within the rosette structure ${ }^{19}$. Close to and within the metamaterial the local field is weak at the center of the rosette pattern, most of the field's energy is found in the vicinity of the outer parts of the rosette arms and in the unstructured dielectric areas at the edge of the unit cell. The field itself develops a complex structure, which can be understood in terms of two regimes. In the ultimate vicinity of each rosette the local field is dominated by the presence of the nearby metal structure, which leads to a local electric field linearly polarized perpendicular to the metal wire. Due to each rosette's four-fold rotational symmetry, however, this alone cannot affect the far-field polarization state. It is the coupling between both mutually twisted metal patterns, that makes the metamaterial polarization sensitive and allows it to rotate the far-field polarization state. Locally this can be seen from the complex field structure in regions that experience substantial field contributions from both rosettes. Here, depending on the phase delays and magnitudes of excited currents, left-handed and right-handed local fields with rotated azimuth are excited. Notably, despite the complex structure of the local fields, azimuth and ellipticity of the effective field typically change continuously along the propagation direction. For the transmitted wave, the complicated substructure of the local field interferes away within about the first $4 \mathrm{~mm}$ of the metamaterial's surface, leaving a rotated elliptical plane wave which propagates to the far-field.

\section{MULTI-LAYERED METAMATERIALS: ULTRA-THIN ROTATORS AND CIRCULAR POLARIZERS.}

Exceptionally large rotary power and circular dichroism for the bilayered structure suggest that multi-layered forms of the metamaterial may have very promising gyrotropic properties. Fig. 6 shows the evolution of transmission properties with increasing number of layers of mutually twisted planar metal rosettes. Transmission levels are presented for both circular polarizations and polarization azimuth rotation is shown for linear polarization.

It is evident that a single layer of metal rosettes on a dielectric substrate (top graphs) does not lead to any significant gyrotropy, even though the metamaterial is topologically $3 \mathrm{D}$-chiral, as the rosettes are placed on only one side of the substrate. A minimum of 2 layers of rosettes is required to achieve strong gyrotropy (see detailed discussion above). In the mono-layered case we observe two iso- 
(a)

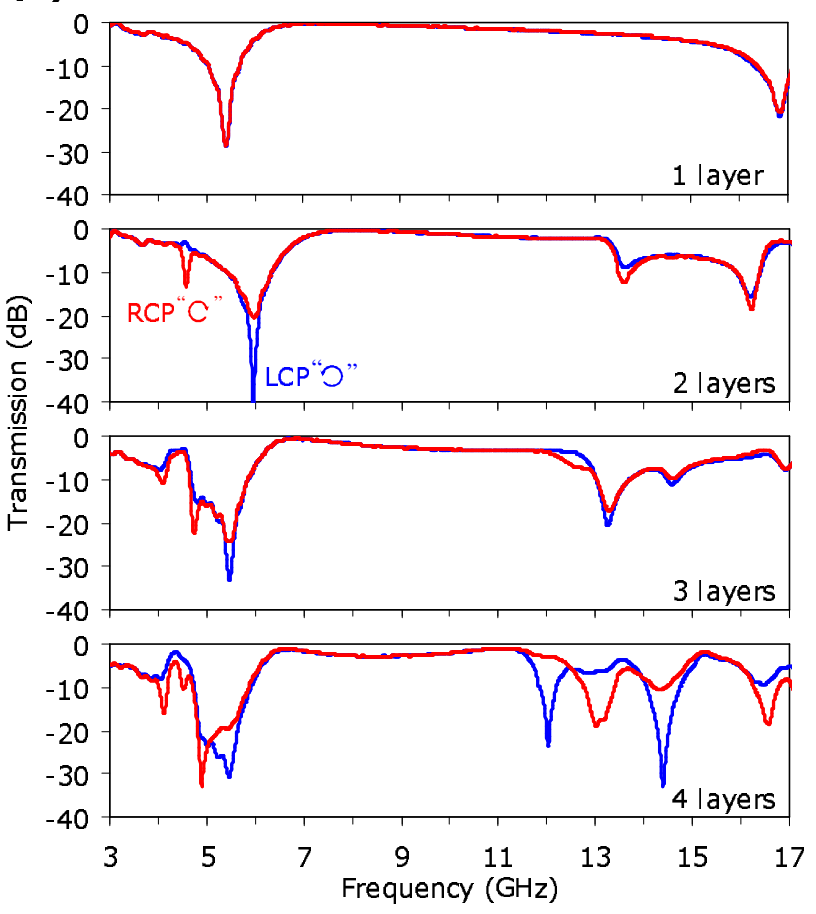

(b)

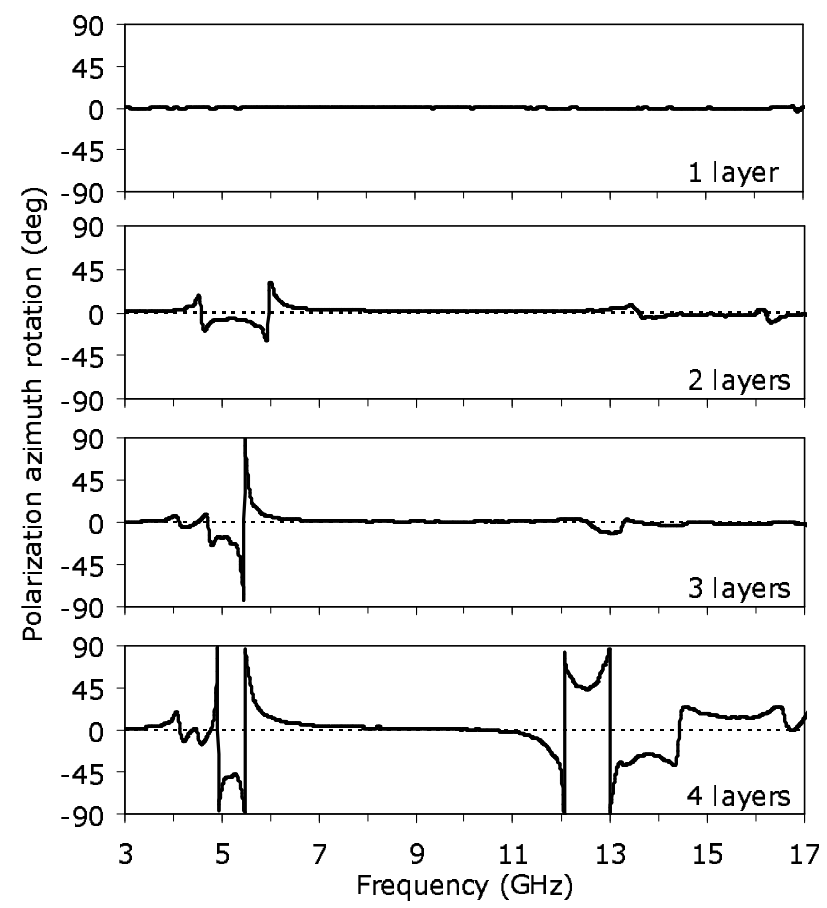

FIG. 6: Development of the metamaterials' transmission characteristics when increasing the number of layers from 1 to 4 . (a) Transmission spectra measured for the metamaterials' circular eigenpolarizations LCP (blue, -) and RCP (red, +). (b) Polarization azimuth rotation measured for linear polarization.

lated resonances corresponding to $\lambda / 2$ and $3 \lambda / 2$ electric dipole excitations. No significant magnetic mode can be excited and therefore gyrotropy is negligible. The introduction of a second layer changes this situation dramatically, by allowing the resonances to split into symmetric (electric) and anti-symmetric (magnetic) current modes excited in pairs of rosettes. The structure's strong gyrotropic response results from the simultaneous scattering contributions of electric and magnetic modes excited in the 3D-chiral structure.

The response of a 3-layered version of the metamaterial is quite similar to the bilayered case, especially in terms of the magnitude of the gyrotropic behavior. However, the resonances split 3 -fold compared to 2 -fold for the bilayered metamaterial.

For 1 to 3 layers, RCP and LCP resonances always occur at the same frequency. Importantly this is not true for 4 or more layers, where we find that the higher-frequency RCP and LCP resonances are spectrally shifted. This allows the 4-layered metamaterial to be almost transparent for one circular polarization while being opaque for the other, making it an efficient circular polarizer. For example at $12 \mathrm{GHz}$ transmission losses are only $3 \mathrm{~dB}$ for RCP but substantial $23 \mathrm{~dB}$ for LCP, leading to a contrast of $20 \mathrm{~dB}$. Between resonances, where transmission levels are equal for both circular polarizations, losses are relatively low while the material's rotary power can be very large. Here the metamaterial shows true optical activity, as it will only rotate the azimuth of the polariza- tion state, without changing its ellipticity. Particularly at $12.5 \mathrm{GHz}$ large absolute rotation of $45^{\circ}$ is achieved with relatively low losses of less than $6 \mathrm{~dB}$ and without changing the polarization state's ellipticity. It must be noted that these functionalities are achieved by a metamaterial that is only $\lambda / 5$ (at $12 \mathrm{GHz}$ ) in thickness, whereas conventionally rotators and circular polarizers are large components many wavelengths in size. The combination of small size, large gyrotropic effects and relatively low losses makes multi-layered forms of the metamaterial suitable for practical use as ultra-thin rotator and circular polarizer.

\section{SUMMARY}

In summary we have realized a class of 3D-chiral metamaterials with very versatile properties including negative index of refraction due to chirality, negative permeability, negative permittivity, giant optical activity and very large circular dichroism. We have given evidence that for our bilayered metamaterial, which is based on mutually twisted planar metal rosettes in parallel planes, the negative refractive index does indeed result from the $3 \mathrm{D}$-chiral nature of the metamaterial and not from driving permeability and permittivity negative in the same frequency range as for conventional negative index media. We have illustrated in terms of local fields how our highly gyrotropic structure interacts with electromag- 
netic waves. And finally we found that multi-layered versions of the metamaterial show enhanced performance, not only in terms of larger circular dichroism and polarization rotation, but also in terms of reduced losses, making them suitable for use as ultra-thin circular polarizers and polarization rotators in practical applications.

\section{Acknowledgments}

The authors at University of Southampton acknowledge financial support of the Engineering and Physical Sciences Research Council, UK under the Nanophotonics Portfolio Grant.

Work at Ames Laboratory was supported by the Department of Energy (Basic Energy Sciences) under contract No. DE-AC02-07CH11358. This work was partially supported by the AFOSR under MURI grant (FA955006-1-0337), by DARPA (Contract No. MDA-972-01-20016), the Office of Naval Research (Award No. N0001407-1-0359) and the European Community FET project PHOME (Contract No. 213390).

The author Jianfeng Dong gratefully acknowledges support of the W.C. Wong Education Foundation, Hong Kong, the National Basic Research Program (973) of China (Grant No. 2004CB719805) and the National Natural Science Foundation of China (Grant No. 60777037).

\section{APPENDIX}

\section{A. Sample description and manufacturing.}

The metamaterial is double-periodic with a square unit cell of $15 \times 15 \mathrm{~mm}^{2}$ (see Fig. 1), which ensures that the structure does not diffract electromagnetic radiation for frequencies lower than $20 \mathrm{GHz}$. The overall size of the samples was approximately $220 \times 220 \mathrm{~mm}^{2}$. The metamaterial's unit cell contains coaxial planar copper rosettes of 4-fold symmetry in parallel planes, which are separated by very thin $(1.6 \mathrm{~mm})$ dielectric layers. Here we present results for four different forms of the metamaterial, corresponding to unit cells containing $1,2,3$ and 4 coaxial rosettes. A mutual anti-clockwise twist of $15^{\circ}$ introduced between adjacent rosettes makes the unit cells containing more than one rosette $3 \mathrm{D}$-chiral. The metamaterial has been manufactured by lithography using standard FR4 circuit board substrates with a dielectric constant of $\varepsilon \approx 4.5+0.15 i$.

\section{B. Measurement technique.}

All transmission and reflection measurements were performed in an anechoic chamber in the $3-17 \mathrm{GHz}$ range of frequencies using broadband horn antennas (Schwarzbeck BBHA 9120D) equipped with lens concentrators and a vector network analyzer (Agilent E8364B).

\section{Effective parameter retrieval.}

For normal incidence, the rosette structure can be modeled as a reciprocal bi-isotropic medium and the constitutive equation is given by

$$
\left(\begin{array}{l}
\mathbf{D} \\
\mathbf{B}
\end{array}\right)=\left(\begin{array}{cc}
\varepsilon_{0} \varepsilon & \mathrm{j} \kappa / c_{0} \\
-\mathrm{j} \kappa / c_{0} & \mu_{0} \mu
\end{array}\right)\left(\begin{array}{c}
\mathbf{E} \\
\mathbf{H}
\end{array}\right)
$$

where $\varepsilon_{0}, \mu_{0}$ and $c_{0}$ are the permittivity, permeability and the speed of light in vacuum, respectively. The eigen solutions in bi-isotropic media are right-handed (RCP) and left-handed (LCP) circularly polarized plane electromagnetic waves. The refractive index for RCP and LCP is given by $n_{ \pm}=\sqrt{\varepsilon \mu} \pm \kappa$, where $(+)$ and $(-)$ denote RCP and LCP. From the complex transmission and reflection coefficients, $T$ and $R$, the refractive index, $n$, and the impendence, $z$, can be obtained ${ }^{18}$,

$$
\begin{aligned}
z & =\sqrt{\frac{(1+R)^{2}-T_{+} T_{-}}{(1-R)^{2}-T_{+} T_{-}}} \\
n_{ \pm} & =\frac{-\mathrm{j}}{k_{0} d} \ln \left[\frac{1}{T_{ \pm}}\left(1-\frac{z-1}{z+1} R\right)\right]
\end{aligned}
$$

where $k_{0}$ is the wave vector in vacuum and $d$ is the thickness of the rosette structure. The branches of the square root and the logarithm function in the formula above have to be chosen carefully according to the energy conservation, i.e. the real part of the impendence is positive, $\operatorname{Re}(z)>0$, and the continuity of $n_{ \pm}$versus the frequency. Finally, for other material parameters, we derive $\kappa=\left(n_{+}-n_{-}\right) / 2, \mu=z\left(n_{+}+n_{-}\right) / 2$ and $\varepsilon=\left(n_{+}+n_{-}\right) / 2 z$.

\footnotetext{
* Email: erp@orc.soton.ac.uk; Homepage: www . nanophotonics.org.uk/niz/

1 D. L. Jaggard and N. Engheta, Electron. Lett. 25, 173 (1989).

2 Y. Svirko, N. Zheludev, and M. Osipov, Appl. Phys. Lett. 78, 498 (2001).

3 A. Lakhtakia and R. Messier, Sculptured Thin Films: Na-
}

noengineered Morphology and Optics (SPIE Press, 2005).

4 G. Shvets., Appl. Phys. Lett. 89, 141127 (2006).

5 J. B. Pendry, Science 306, 1353 (2004).

6 S. Tretyakov, I. Nefedov, A. Sihvola, S. Maslovski, and C. Simovski, J. Electromagn. Waves Appl. 17, 695 (2003).

7 S. Tretyakov, A. Sihvola, and L. Jylhä, Photonics Nanostruct. Fundam. Appl. 3, 107 (2005). 
8 Y. Jin and S. He, Opt. Express 13, 4974 (2005).

${ }^{9}$ C. Monzon and D. W. Forester, Phys. Rev. Lett. 95, 123904 (2005).

10 Q. Cheng and T. J. Cui, Phys. Rev. B 73, 113104 (2006).

11 V. M. Agranovich and Y. N. Gartstein, Physics-Uspekhi 49, 1029 (2006).

12 A. V. Rogacheva, V. A. Fedotov, A. S. Schwanecke, and N. I. Zheludev, Phys. Rev. Lett. 97, 177401 (2006).

13 E. Plum, V. A. Fedotov, A. S. Schwanecke, N. I. Zheludev, and Y. Chen, Appl. Phys. Lett. 90, 223113 (2007).

14 D. R. Smith, W. J. Padilla, D. C. Vier, S. C. Nemat-Nasser, and S. Schultz, Phys. Rev. Lett. 84, 4184 (2000).
15 G. Dolling, C. Enkrich, M. Wegener, C. M. Soukoulis, and S. Linden, Science 312, 892 (2006).

16 O. Paul, C. Imhof, B. Reinhard, R. Zengerle, and R. Beigang, Opt. Exp. 16, 6736 (2008).

17 R. D. Hollinger, V. V. Varadan, D. K. Ghodgaonkar, and V. K. Varadan, Radio Sci. 27, 161 (1992).

18 T. Koschny, J. Zhou, and C. M. Soukoulis, under preparation (2008).

19 The effective polarization state is the polarization of the average field in a plane perpendicular to the propagation direction. 